\title{
Stärken und Schwächen ordonomischer Überbietungsargumente
}

\section{Kommentar zum Hauptbeitrag von Ingo Pies}

[1] Ingo Pies stellt in seinem Übersichtstext »Ordonomik als Methode zur Generierung von Überbietungsargumenten « das beeindruckend komplexe Ensemble von Ideen vor, die das Forschungsprogramm der Ordnungsethik bzw. Ordonomik prägen. Diese Komplexität kann ich im Rahmen dieses kleinen Kommentars nicht würdigen, stattdessen konzentriere ich mich auf zwei Kritikpunkte: einen allgemeinen Vorbehalt und einen besonderen Einwand.

[2] Die Ordonomik ist als eine differenzierte Verantwortungsethik zu begreifen. Verantwortung wird in der Ordonomik über drei analytisch zu unterscheidende Ebenen (auch ssocial arenas genannt) verteilt gedacht: die Ebene des Basis-Spiels (๖basic game`), wo die Interaktionen von (natürlichen personalen, aber auch künstlichen: kollektiven, korporativen) Akteuren von statten gehen, zweitens die des Meta-Spiels, wo die Institutionen in Form von konstitutiven und organisationalen Regeln ihre soziale Wirklichkeit haben, und schließlich die des Meta-MetaSpiels, die Sphäre der Ideen, des Gedanklichen, des Normativen und der semantisch bedeutungsvollen sprachlichen Äußerungen. Im Rahmen der Ordonomik sind diese drei Ebenen oder Arenen (die, darauf legt Pies Wert, in keinem Sinne >ontologisch`, sondern nur sschematisch verstanden werden sollen - was immer das heißen mag) als ein Kreisprozess der wechselseitigen Korrekturen und Anpassungen und so als ein Lernprozess zu denken: »the meta-meta game restricts the meta game via normative expectations, and the meta game restricts the basic game via institutional incentives. Looking at it the other way round, i.e. bottomup, there are important feedback mechanisms from lower to higher levels without which societal learning processes could not succeed « (Pies 2016: 150).

[3] Ich meine: Wenn die Ordonomik Kreisprozesse als Lernprozesse innerhalb des Zusammenspiels der drei `Spiele bzw. der drei Arten von Spielen denkt, dann erfordert das geeignete Bewertungsmaßstäbe bzw. -standards, um zwischen Verschlechterungen und Verbesserungen und beide von bloßen Veränderungen zu unterscheiden. Tatsächlich lokalisiert Pies solche Maßstäbe bzw. Standards innerhalb der `normativen Semantik ‘ des Meta-Meta-Spiels - und berücksichtigt so die m.E. normativitätstheoretisch wertvolle Entdeckung der Homann-Schule, dass die praktischen Bedingungen, unter denen normative Ideen in Interaktionen übersetzt werden sollen und können, den Geltungsanspruch und den Inhalt der betreffenden normativen Ideen nicht absolut indifferent lassen, sondern auf ihn zurückwir-

* Prof. Dr. Matthias Kettner, Fakultät für Kulturreflexion, Universität Witten/Herdecke, Alfred-Herrhausen-Straße 50, D-58448 Witten. Tel.: +49-(0)2302-926811, E-Mail: kettner@uni-wh.de, Forschungsschwerpunkte: Diskursethik, Sozio-kulturelle Pathologien, Wirtschaftsphilosophie. 
ken: das zumutbare Können restringiert das sinnvolle Sollen nicht nur (ein altes robustes Rationalitätsprinzip der praktischen Philosophie: Sollen impliziert Können), sondern modifiziert es sogar. Soweit, so gut. Doch die stärksten, letztlich maßgeblichen und das Anpassungsgeschehen insgesamt bestimmenden QuasiStandards lokalisiert Pies nicht mehr innerhalb der normativen Arena, sondern er denkt sie als objektive und, relativ zum Eigensinn der normativen Sphäre gesehen, gewissermaßen externe: es sind die Erfordernisse >der modernen Gesellschaft in allem, was für die Selbsterhaltung der herrschenden gesellschaftlichen Verhältnisse funktional notwendig ist.

[4] Gewiss, der modale Gedanke, dass die Selbsterhaltung von $\mathrm{x}$ in aller möglichen Kritik an $\mathrm{x}$ der Kritik entzogen bleiben müsse, enthält als solcher keinen Widerspruch. Doch wieviel Vernünftiges an diesem Begrenzungsgedanken daran ist, hängt ganz davon ab, wie man das gemeinte x genauer bestimmt. Auch in der Diskursethik stützen wir uns auf ihn, wenn wir argumentieren, dass die Selbsterhaltung der sinnvollen Dialogpraxis des argumentativen Diskurses alle diejenigen logischen und moralischen Normen, die für diese Praxis konstitutiv sind, der Kritik entzieht, da jede Kritik, wenn und indem sie sinnvoll ist, diese Normen bereits in Anspruch nimmt und bestätigt. Die Ordonomik scheint an die Stelle von x rdie moderne Gesellschaft< einzusetzen. Die Ordonomik enthält aber (noch) keine sozialtheoretisch wirklich reichhaltige Analyse der Modernität moderner Gesellschaften, sondern bestimmt letztere auf der schmalen Basis von nur zwei Merkmalen: Wettbewerb und Wertepluralismus. Dadurch - und das ist mein erstes Bedenken - schränkt die Ordonomik, soweit sie sich als Ethik versteht, ihre Kraft moralischer Kritik ein: Sie will alle moralische Anforderungen, an den herrschenden gesellschaftlichen Verhältnissen etwas zu verändern oder aber zu bewahren, genau nur so weit sinnvoll finden, wie diese nicht systemkonstitutiv sind. Sie will als Wirtschaftsethik »dem Wettbewerbselement in der modernen Wirtschaft - und allgemein: dem Wettbewerbselement in allen Funktionssystemen der modernen Gesellschaft, insbesondere in Politik, Öffentlichkeit, Zivilgesellschaft und Wissenschaft - systematisch Rechnung tragen « ([6]). Ich meine: Positionsintern wird der Wettbewerb so in den Rang eines unbedingt Erhaltenswerten gesetzt, an dem moralische Kritik ihr Ende findet. Die Ordonomik ist an ihrer ethischen Wurzel konservativ, nämlich marktwirtschaftskonservativ. Um dies mit einem Begriff von Charles Taylor auszudrücken: Den Ordonomen gilt Wettbewerblichkeit als ein Hyper-Gut im Raum moralischer Orientierungen moderner Gesellschaften. Aber warum sollten wir alle den Ordonomen hierin folgen?

[5] Mein besonderer Einwand betrifft den im Werkzeugkasten der Ordonomik prominenten Begriff der >orthogonalen Positionierung . Methodisch zielt diese Operation darauf ab, die Wahrnehmung bzw. Deutung von und dadurch den Umgang mit Wertkonflikten, die innerhalb von spezifisch moralischen Diskursen oder überhaupt in normativen Diskursen jeglicher Art auftreten, beherzt zu verrücken: Soweit wie möglich weg vom Deutungsmuster eines Nullsummenspiels (N), so nahe wie nötig heran an das Deutungsmuster von Win-Win-Spielen (W-W). Nota bene: Den Gedanken, dass W-W ceteris paribus besser, nämlich für alle Beteiligten vorzugswürdig sind im Vergleich zu N, nimmt Pies als eine nicht weiter 
begründungsbedürftige Prämisse an. Ich hingegen möchte an seiner Fragwürdigkeit und insofern Begründungsbedürftigkeit festhalten. Das spieltheoretische Schema W-W vs. N funktioniert nur mit der nichttrivialen werttheoretischen Voraussetzung, dass alle Werte, auf die sich das Schema erstreckt, vollständig miteinander kommensurabel sind. Daher muss in jedem Fall der Anwendung des Schemas begründet werden, dass diese axiologische Bedingung für die betreffende soziale Wirklichkeit, die man mit Hilfe des Schemas besser zu begreifen hofft, erfüllt ist und die Schematisierung allen in der betreffenden sozialen Wirklichkeit wirksamen Werten gerecht wird, sie also nicht reduziert oder auf andere Weise entstellt.

[6] Mit Hilfe der fälschlich für unmittelbar evident gehalten normativen und evaluativen Prämisse (= W-W ist besser als $\mathrm{N}$ und sollte daher angestrebt werden) kann sich die Ordonomik die diskursive Last von eigenständigen ethischen Begründungen für spezifische ordonomische normative Maßstäbe und Standards (z.B. moralische Normen) ersparen und als positive Wissenschaft auftreten, die hypothetischen politischen Rat zu geben vermag: »the ordonomic approach aims at generating positive analyses that inform and improve normativity without taking sides in a controversial value conflict « (Pies 2016: 152). Das klingt so, als könne die Ordonomik wohlbegründete Interventionen in Konflikte vorschlagen, ohne Partei ergreifen zu müssen. Zu schön, um wahr zu sein. Denn zugleich fordert die Ordonomik qua wirtschaftsethische Position, dass Ordonomen alle Bürger dazu anhalten sollten, eine gewisse Art von Mit-Verantwortung (>OrdoResponsibility`) für die Verbesserung der Verhältnisse zu übernehmen, dahingehend, dass im allgemeinen Win-Win-Situationen wahrscheinlicher werden: »the ordonomic approach addresses all persons that are capable of participating in meta games and meta-meta games and tries to encourage them to actively take >ordo responsibility in order to system(at)ically improve the outcomes of basic games « (ebd.; kursive Hervorhebung durch MK).

[7] Aber könnte man die soeben zitierte Selbstauskunft der Ordonomik nicht doch auch normativitätsfrei verstehen? Einfach als Tatsachenfeststellung, was Ordonomen tun? Vielleicht. Allerdings wüsste man dann doch gerne, was Ordonomen tun sollten und auch, warum sie meinen, tun zu sollen, was sie tun - Fragen des normativen Selbstverständnisses einer Disziplin, der sich keine umsichtige Ökonomiken unbeschadet entziehen kann, wie wir spätestens seit Buchanans What Economists Should Do wissen. So gesehen bleibe ich bei meiner Problemdiagnose: Die Ordonomik macht als angewandte Ethik diskursiv verhakten antagonistischen Meinungslagern ein Interventionsangebot (`orthogonale Positionierung ), die Problemlage doch bitte nicht mehr nach Schema $\mathrm{N}$ zu sehen, sondern vernünftigerweise nach Schema W-W. Sie präsentiert ihr Interventionsangebot als ein sachlich vernünftiges, ansonsten unparteiliches, weil allparteiliches (und eigentlich: unwiderstehliches) Angebot. Ich halte diese All- und Unparteilichkeit und insofern Seitenneutralität für ein Stück ordonomische Ideologie: Die dem Forschungsprogramm Ordonomik interne normative Ethik fordert diese ordonomische Intervention und sie erfordert zugleich, die ordonomische Intervention für unparteilich zu halten. Genauer: sie fordert, die Beteiligten und Betroffenen (egal 
ob sie Ordonomen sind) aufzufordern, die Dinge so zu sehen (nach Schema WW). Wer nun soll auffordern? Wenn die Ordonomen hier nicht selbst in die Verantwortung eintreten wollen, wodurch sie unweigerlich Partei im Streitfeld selber würden, müssen es wohl die Beteiligten und Betroffenen selber sein, die einander auffordern, die also von sich selbst und voneinander fordern, sich orthogonal zu positionieren (um die Dinge nach Schema W-W zu sehen). Die hierin liegende normative Zumutung an alle Beteiligten und Betroffenen müsste aber diesen selber gegenüber von den Ordonomen gerechtfertigt werden; ob und wie weit das im jeweiligen Konfliktfeld gelingt, kann nur und muss ich sich in realen Diskursen erweisen. Andernfalls betreiben die Ordonomen question begging. Das kann man so machen, ist aber philosophisch suboptimal.

[8] Der Eindruck des question begging der Ordonomen drängt sich auf, wenn man sieht, dass die Ordonomik allgemein allen Beteiligten und allen Betroffenen gleichermaßen ein vergleichbares Interesse unterstellt, »to system(at)ically improve the outcomes of basic games " (s.o.). Was, wenn überhaupt, berechtigt sie zu dieser allgemeinen Unterstellung? Die Unterstellung bleibt so formal, wie es eine Moral wäre, deren allgemeine Forderung wäre, das Gute zu suchen und das Schlechte zu meiden: man nickt, aber die Forderung ist allgemein zustimmungsfähig nur so lange, wie sie leer bleibt. Im konkreten Fall wird sie sofort strittig, weil die beiden axiologischen Leerstellen von verschiedenen Akteuren verschieden ausgefüllt werden. Wie oben in Absatz [5] zum N vs. W-W Schema bemerkt, ist es keineswegs selbstverständlich - und sollte angesichts der Betonung von Wertepluralismus für Ordonomen noch viel weniger selbstverständlich sein -, dass Beteiligte und Betroffene unter outcomes, die es zu verbessern gilt, dasselbe verstehen, also die Dinge im Licht kommensurabler Werte interpretieren und bewerten. Die Ordonomik macht ein Interventionsangebot, das nach Maßgabe ihrer eigenen Annahmen nicht nur question begging, sondern auch wenig erfolgversprechend ist.

[9] Die Argumentform, mit der die Ordonomik für ihr Interventionsangebot der orthogonalen Positionierung den sanften Zwang des besseren Arguments ausüben will, nennt Pies »Überbietungsargumente «: Argumente, die ihre Adressaten rational motivieren können sollen, sich zu wertstrittigen Trade-off-Vorstellungen orthogonal zu positionieren, also vom N-Schema weg und zum W-W-Schema hinzukommen. Sie sollen Diskursversagen therapieren. Diskursversagen liegt vor, wenn Diskussionsteilnehmer »eine radikale Aufteilung in zwei Lager « ([14]) vornehmen - im besonderen Falle von politisch diskutierenden Öffentlichkeiten eine Aufteilung "pro und contra Regierungspolitik. In beiden Fällen halten die extremen Kontrahenten jeweils sich selbst für moralisch gut, während sie ihren Gegenpart als unmoralisch einstufen " (vgl. [14]). »Wer sich selbst für 'gut ‘ hält und die andere Seite für unmoralisch, hört nicht mehr zu und spricht lieber über die andere Seite als mit ihr. Diese Form der Auseinandersetzung verbindet nicht. Sie spaltet. Der Diskurs versagt « ([17]).

[10] Ich stimme Pies zu, dass zur Verbesserung der Diskursfähigkeit in Kommunikationsgemeinschaften alles erforderlich ist, was es braucht, damit trotz tiefer Meinungsverschiedenheiten die egalitäre Anerkennung zumindest als gleichbe- 
rechtigte Diskurspartner generalisiert wird. Die Auffassung der Ordonomik deckt sich hier mit der einer realistischen Diskursethik: Ohne egalitäre Anerkennung keine reale Diskursivität; die Grenzen der ersteren sind auch die Grenzen der letzteren (vgl. Kettner 2016). Aber Pies erklärt Diskursversagen m.E. zu eng, wenn er »die Ursache des Problems in einer dysfunktionalen (Ver-)Wendung der Moralkommunikation « ([45]) ordonomisch erklärt. Moralische, aber auch viele andere, z.B. politische und vor allem religiöse identitätsrelevante Richtigkeitsüberzeugungen können Kommunikationsgemeinschaften diskursiv fragmentieren, so dass Kommunikation zwar noch in vielen Formen erfolgen kann (z.B. in Formen der Hass-Rede oder in Formen des Ignorierens), aber nicht mehr in der bestimmten Form ernsthaften Argumentierens.

[11] Die Ankündigung eines neuen Typs von Argumenten war und bleibt immer eine philosophische Sensation (man denke an stranszendentale Argumente). Was macht »Überbietungsargumente « zu solchen? Überbietend im Sinne der Ordonomik argumentiert man, »indem zunächst beide Positionen « von diskursiv verhakten antagonistischen Meinungslagern relativ zu einer Problemlage »dekonstruiert und sodann im Zusammenhang rekonstruiert (und argumentativ überboten) werden « ([46]). Überbietungsargumente müssen als gleichstarkes, aber inhaltlich gegensätzliches Paar entwickelt werden. Es sind »die dem Trade-off-Denken zugrunde liegenden normativen Anliegen ernst zu nehmen, um sie in der Moraldimension ihres je eigenen Anspruchs zu übertreffen " ([61]).

[12] Um das Beispiel »Flüchtlings(politk)debatte « aufzugreifen, das Pies dankenswerterweise konkret ausgearbeitet hat (vgl. [43]- [55]):

Angenommen, man seziert die vielfältigen verhakten antagonistischen Meinungslager relativ zu Problemlagen des Politikfelds Flüchtlingspolitik entlang einer Trennlinie, die durch zwei als moralisch relevant wahrgenommene Ansprüche gegeben ist, in zwei Positionslager P1 und P2, so identifiziert man mit Pies P1 durch den Anspruch, durch humanitäre Hilfe »solidarisch « ([62]) zu handeln und P2 durch den Anspruch, durch Kontrolle von Zuwanderung »dem nationalen Interesse der Deutschen « (ebd.) dienlich zu sein.

Eine Überbietungsargumentation soll dann adressiert an P1 zeigen, dass durch humanitäre Hilfe solidarisch zu handeln, so gut das ist, doch auch moralische Kosten hat, ${ }^{1}$ die durch eine alternative Handlungsweise $\mathrm{H}$ gesenkt werden könnten: P1, ihr könnt es besser machen! Mutatis mutandis für P2. Im dritten Schritt der Überbietungsargumentation versucht der Ordonom, aus dem an P1 und P2 gerichteten »ihr könnt das besser « ein »ihr könnt es gemeinsam besser « zu machen: eine Synthese, in der die jeweiligen Anliegen von P1 und P2 zum Zuge kommen würden, aber nicht mehr auf Kosten von einander.

[13] Hier sehe ich u.a. folgendes Problem: Im Zuge der Entwicklung von $\mathrm{H}$ muss der Ordonom nicht nur das Anliegen von P1 setzen - schon das ist hinterfragbar, denn in puncto Flüchtlingspolitik wird sich der Raum konfligierender, aber von den Betreffenden je als gut wahrgenommener Gründe in weit mehr als nur zwei Positionslager zerlegen -, sondern auch fortlaufend reinterpretieren, z.B.

1 Zum Begriff von moralischen Kosten siehe Kettner 2014. 
als das, "weltweit möglichst vielen Menschen wirksam zu helfen«([48]). Letzteres ist ersichtlich eine utilitaristisch anmutende, also spezielle Version des humanitären Hilfegebots, und vermutlich würden nicht alle, die man zuvor als das Lager P1 geclustert hat, ihr Anliegen darin wiedererkennen können. Ähnlich für P2.

[14] Die Problematik, dass vielfältige Anliegen vielfältige Trennlinien schaffen und dass die Überbietungsargumentation, indem sie rekonstruieren und dekonstruieren muss, strittige Anliegen und deren Interpretationen verändert und dadurch unter Umständen sogar neue und mehr Trennlinien schafft, belastet dann auch den dritten Schritt der ordonomischen Überbietungsargumentation. Pies meint für den konkreten Fall: »Ein beiden Seiten gemeinsames Anliegen besteht perspektivisch darin, die Mittelmeerroute zu schließen und gleichzeitig - als Junktim! - ein geordnetes Verfahren zu etablieren, das politisch Verfolgten Asyl bietet, Flüchtlingen das Leben rettet sowie für Kontingente von Arbeitsmigranten und ihre Familien eine legale Option eröffnet, ohne Lebensgefahr nach Europa zu kommen « ([55]). Ich bin nicht überzeugt, dass dieser Vorschlag mehr Konsens zwischen P1 und P2 (und P3...Pn) schafft, als Dissens. - Die Denkfigur der Überwindung antagonistischer Positionen durch Überbietung ist wichtig und interessant. Alternativen zu ihr wären zu erwägen, z.B. die dialektische Überwindung. Hier müsste man jeder Seite die innere Widersprüchlichkeit aufzeigen. Interessant wäre auch ein Vergleich mit Versuchen wie dem von Ronald Dworkin, die Diskursblockade in der amerikanischen Abtreibungsdebatte aufzuheben (vgl. Dworkin 1994).

\section{Literaturverzeichnis}

Dworkin, R. (1994): Life's Dominion. An Argument about Abortion, Euthanasia, and Individual Freedom, New York: Vintage Books.

Kettner, M. (2014): Wann haben wir ein moralisches Problem? In: Maring, M. (Hg.): Bereichsethiken im interdisziplinären Dialog, Karlsruhe: KIT Scientific Publishing, 25-44.

Kettner, M. (2016): Der Raum der Gründe und die Kommunikationsgemeinschaft der Begründer, in: Deutsches Jahrbuch für Philosophie, Bd. 5, Hamburg: Felix Meiner, 2016, 637655.

Pies, I.: Taking or Locating Responsibility? In: Zeitschrift für Wirtschafts- und Unternehmensethik, Jg. 17/H. 1, 148-153. 